\title{
Nonalcoholic fatty liver disease and type 2 diabetes: where do Diabetologists stand?
}

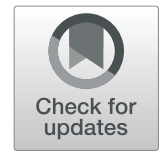

Shaheen Tomah $^{1,2^{*}}$ (D) Naim Alkhouri ${ }^{3}$ and Osama Hamdy ${ }^{1,2}$

\begin{abstract}
Background: Nonalcoholic fatty liver disease (NAFLD) is the most common chronic liver disease worldwide. The increasing prevalence of NAFLD mirrors that of obesity and type 2 diabetes over the last two decades.

Main: In a two-way pathophysiologic relationship, NAFLD increases the risk of developing type 2 diabetes, while the latter promotes the progression of simple fatty liver to a more advanced form called nonalcoholic steatohepatitis (NASH). NASH increases the risk of cirrhosis and hepatocellular carcinoma (HCC), which may require liver transplantation. With the absence of FDA-approved medications for NAFLD treatment, lifestyle intervention remains the only therapy. Lately, extensive research efforts have been aimed at modifying NASH fibrosis and developing noninvasive screening methods.
\end{abstract}

Conclusion: We highlight the pathophysiologic relationships between NAFLD and type 2 diabetes, discuss disease recognition, models of care, and current and emerging therapies for NASH treatment.

Keywords: Type 2 diabetes, Nonalcoholic fatty liver disease, Nonalcoholic steatohepatitis, Pathophysiology, Awareness, Screening, Treatment

\section{Background}

Nonalcoholic fatty liver disease (NAFLD) is an umbrella term that encompasses multiple progressive liver disorders, ranging from simple hepatic steatosis, often called nonalcoholic fatty liver (NAFL), to nonalcoholic steatohepatitis (NASH) which is marked by hepatocyte inflammation and ballooning. Around 35\% of NASH cases progress to liver fibrosis [1] and potentially to end-stage liver disease or hepatocellular carcinoma (HCC) $[2,3]$. The growing epidemic of NAFLD in western societies is estimated to affect around 20 to $30 \%$ of the overall population and 45 to $75 \%$ of patients with type 2 diabetes $[4,5]$. Over the last two decades, the high prevalence rates of NAFLD have been paralleling the rapidly progressing epidemic of obesity and type 2 diabetes [6, 7]. In fact, we see NAFLD and type 2 diabetes at the intersection of similar risk factors, epidemiology, and

\footnotetext{
* Correspondence: shaheen.tomah@joslin.harvard.edu

${ }^{1}$ Research Division, Joslin Diabetes Center, 1 Joslin Place, Boston, MA 02215, USA

2Department of Medicine, Harvard Medical School, Boston, MA 02215, USA Full list of author information is available at the end of the article
}

pathophysiology [8-10]. In terms of precedence, the recognition of NAFLD as a major chronic disease is relatively new compared to type 2 diabetes [11]. This is also evident in medical literature over the past 40 years (Fig. 1). Currently, NAFLD is considered the most common chronic liver disease worldwide and a leading etiology of liver diseases among adults awaiting liver transplantation in the US [5, 12-14]. The co-existence of NAFLD and type 2 diabetes significantly increases the likelihood of developing NASH and cirrhosis compared to the presence of NAFLD without persistent hyperglycemia [10]. The involvement of NAFLD as an independent predictor of cardiovascular disease (CVD) events remains debatable $[2,15,16]$. Interestingly, the highest mortality in NAFLD is attributed not to endstage liver disease, or risk of HCC, but to worse CVD risk profile [17] possibly driven by the comorbidity of type 2 diabetes and other established CVD risk factors [15]. Hence, increased risk of CVD in patients with type 2 diabetes and NAFLD may exert significant impact on their mortality. With recent advances in NAFLD diagnosis and many phase III trials for NASH-specific therapies, there is 


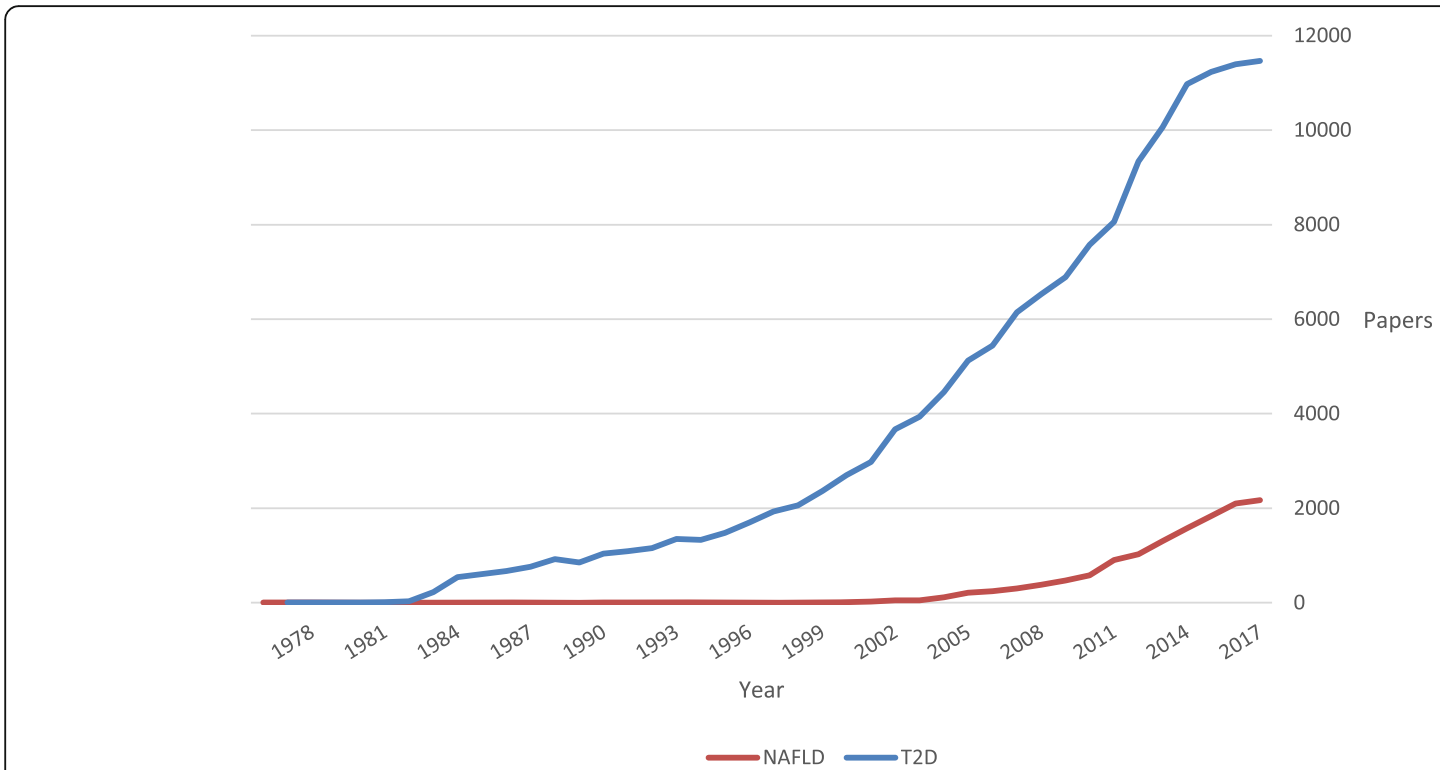

Fig. 1 Nonalcoholic fatty liver disease and type 2 diabetes in publications over four decades. Based on data from Pubmed.gov literature search with the keywords: nonalcoholic fatty liver disease OR type 2 diabetes. Abbreviations: NAFLD, nonalcoholic fatty liver disease; T2D, type 2 diabetes

an essential evolving role for diabetologists in identifying patients with type 2 diabetes at high risk for NAFLD complications and initiating an integrated multidisciplinary plan of care to achieve the best possible results.

In this review, we highlight the pathophysiologic relationships between NAFLD and type 2 diabetes, discuss disease recognition, current therapeutic options, and summarize emerging novel therapies for NASH treatment.

\section{Main text}

\section{Pathogenesis of NAFLD in relation to type 2 diabetes}

The pathogenesis of NAFLD has not been fully unraveled. Footprints of insulin resistance (IR) with associated subclinical inflammation is one of many that were recognized in the course of NAFLD. In this pro-inflammatory state, an increased influx of free fatty acids (FFAs) to the liver causes fatty infiltration in the hepatocytes, which induces liver damage via lipid peroxidation and mitochondrial dysfunction $[18,19]$. Another important source of fatty acids and intrahepatic triglycerides in patients with NAFLD is de novo lipogenesis (DNL), even under fasting conditions, compared to obese patients without NAFLD [20]. Furthermore, obesity per se through adipose tissue inflammation and increased importation of FFAs to the liver has also been considered an important cause of hepatocellular injury [21]. Beyond obesity, chronic glucotoxicity aggravated by persistent hyperglycemia is a key phenomenon observed in the course of type 2 diabetes [22]. Glucotoxicity may promote the progression of NASH via glucose-induced IR, increased DNL, and hepatocellular dysfunction [23]. On the other hand, a recent animal study showed that dietary fructose, but not glucose, impaired fat metabolism via changes in mitochondrial morphology and function when added to a high-fat diet [24]. Many other factors are involved in the pathogenesis of NAFLD comprising mechanisms in the gut, adipose tissue, and liver (Fig. 2). These are often referred to in the literature as the gut-fat-liver axis [25]. Recent advances in multi-omics studies with gut microbiota profiling showed that increased metabolic endotoxemia due to high gut permeability is closely tied to the development and progression of NAFLD [26]. These consecutive or somewhat parallel mechanisms promote cell stress and apoptotic pathways. In NASH, Lipotoxicity-induced hepatocyte ballooning leads to downregulation of a key player in the apoptotic pathway, which is caspase 9, and along with reinforcement from a hedgehog autocrine survival signaling pathway produces an "undead hepatocyte" in which apoptosis has been initiated but fails to be executed driving a vicious pathway of inflammation (NASH) and fibrosis [27]. Among all the histologic features of NASH, fibrosis is the most important predictor of end-stage liver disease and increased mortality [28]. Obesity, metabolic endotoxemia, and IR are all hallmarks of the metabolic syndrome (MetS) and type 2 diabetes [29-31]. Now that NAFLD is considered by many as the hepatic manifestation of the MetS, the magnitude of the problem can be better appreciated [32, 33].

\section{Disease recognition}

The diagnosis of NAFLD could be missed due to the lack of cost-effective, non-invasive diagnostic tools, and the absence of a clear consensus on the value of screening for NAFLD [2,34-36]. A recent large-scale study reported a significant gap in diagnosing NAFLD based on 


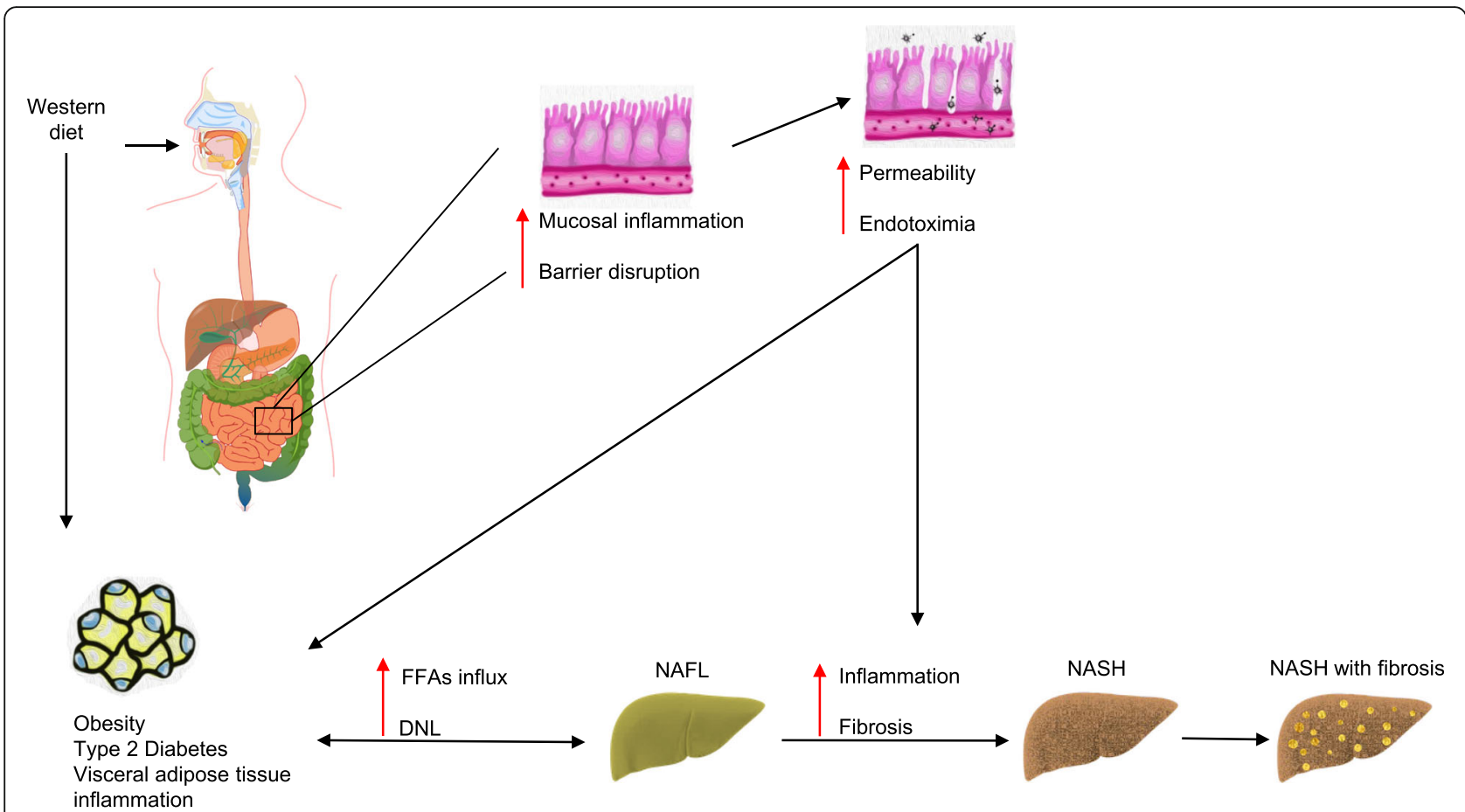

Fig. 2 Key players in the development of NAFLD/NASH comprising mechanisms in the gut, adipose tissue and liver. Abbreviations: FFA, free fatty acid; NAFL, nonalcoholic fatty liver; DNL, de novo lipogenesis; NASH, nonalcoholic steatohepatitis

primary-care records of almost 18 million adults from the UK, Italy, Spain, and the Netherlands [37]. This mandates a multidisciplinary approach aimed to identify patients at risk for developing the advanced form of the disease and accelerate the advancement of research to explore new targets for therapy and reliable serum-based biomarkers. Although it is well-established that patients with type 2 diabetes are at a substantially increased risk for NAFLD complications, patients with type 1 diabetes who are overweight or obese may not be immune. Currently, around $50 \%$ of patients with type 1 diabetes have weight problems; a condition frequently named doublediabetes $[38,39]$. Despite these concerns, it is still debated whether to screen patients with diabetes for NAFLD or not $[2,35]$.

\section{NASH as a complication of type 2 diabetes}

The relationship between NAFLD and type 2 diabetes is bidirectional $[6,40]$. Diabetes promotes the progression of NAFL to NASH and increases the risk of cirrhosis and HCC. On the other hand, NAFLD is associated with an increased risk of developing type 2 diabetes [8-10].

In 1980, Ludwig et al. coined the term "nonalcoholic steatohepatitis" after reporting a case-series of 20 patients with liver histology characterized by fat accumulation and hepatic necroinflammation in the absence of excessive alcohol consumption [41]. Thus, the current terminology of "nonalcoholic fatty liver disease" is mainly derived from excluding alcohol-related heptopathology, focusing on what "does not" lead to this type of fatty liver rather than what "may lead to it." This is mainly due to knowledge gaps in understanding the natural history of NAFLD, which also poses an obstacle to developing a clear approach to the care of patients with co-existing type 2 diabetes [42]. Furthermore, the inclusion of NAFLD among diabetes-related complications is a matter of debate, which is unlikely to be resolved without mechanistic studies that evaluate the relationship between both diseases. Moreover, a discussion about how to define NAFLD in the presence of a pre-existing type 2 diabetes is needed [43]. What is agreed on is that both conditions are related to obesity, subclinical inflammation, and insulin resistance, but the sequence of events is poorly identified.

\section{Noninvasive assessment of NAFLD}

The standards of care of the American Diabetes Association (ADA) recommend evaluating patients with type 2 diabetes or pre-diabetes, who have elevated alanine aminotransferase (ALT) or NAFL by ultrasonography (US) for NASH and liver fibrosis. The ADA guidelines suggest using vibration controlled transient elastography (VCTE) and noninvasive biomarkers for risk-stratification [44]. According to the American Association for the Study of Liver Diseases (AASLD), the diagnosis of NAFLD is defined by the presence of $\geq 5 \%$ hepatic steatosis either by imaging or histology with the absence of secondary 
causes of hepatic steatosis such as high alcohol consumption, monogenic hereditary disorders like Wilson's disease or long-term use of steatogenic medications like methotrexate, amiodarone, and tamoxifen [2]. Liver biopsy remains the gold standard technique for diagnosing $\mathrm{NASH}$ and liver fibrosis; however, it is invasive, carries some intrinsic morbidity and mortality risk, may fail in staging the disease is subject to sampling error, and has reading variability [45].

In clinical practice, US is the recommended first-line imaging technique for diagnosing NAFLD; however, its sensitivity is reduced when hepatic fat content is $<20-33 \%$ [ 46 , 47]. Other non-invasive tools have been developed for diagnosing NAFLD. These include magnetic resonance spectroscopy and magnetic resonance elastography. However, these tools are expensive, time-consuming, and are not considered cost-effective for large-scale NAFLD screening. In clinical research, recent data showed that magnetic resonance imaging-derived proton density fat fraction (MRIPDFF) is a reliable, non-invasive alternative to conventional liver biopsy in assessing response to treatment in earlyphase NASH trials [48]. On the other hand, VCTE is an imaging technology widely used at liver clinics as a simple aid for diagnosis and follow up of patients with NAFLD and other chronic liver diseases [49]. VCTE has the advantage of evaluating a portion of the liver that is 100 -fold greater than that evaluated by needle biopsy and in much shorter time. The generic name for VCTE is Fibroscan ${ }^{\circ}$ (Echosens Paris, France), which produces a quantifiable, reproducible liver stiffness measurement (LSM) expressed in kilopascals $(\mathrm{kPa})$. A LSM value of $>9.8 \mathrm{kPa}$ is consistent with the presence of advanced fibrosis/cirrhosis [50-52]. More recently, the growing interest in precision medicine led to the development of liquid biopsy tools. These are non-invasive, mechanism-based biomarkers that have the potential to eventually replace conventional needle biopsy for diagnosis, stage stratification, and monitoring of response to treatment in NASH and other chronic liver diseases [53]. Table 1 provides a summary of the most reliable and widely used imaging modalities for NAFLD diagnosis.

Many noninvasive scores that are simply calculated using routinely available labs and demographic data have been developed to predict the presence of suspected NAFLD [54, 55] including the hepatic steatosis index (HSI) [56], and fatty liver index (FLI) [57]. Other scores could predict the presence of advanced fibrosis (Table 2) such as the FIB-4 index [60], NAFLD fibrosis score (NFS) [61], the enhanced liver fibrosis (ELF) score [59] and alanine aspartate transferase (AST) to platelet ratio (APRI) [62]. Despite their poor sensitivity in detecting advanced fibrosis in patients with type 2 diabetes [63], these scores (FIB-4 is among best studied) $[64,65]$ have reasonable specificity and can be convenient for healthcare providers to assess patients with suspected NAFLD based on US or elevated levels of ALT [58, 66] (Fig. 3). It is important to recognize that patients with the NAFLD spectrum may still present with normal ALT levels including those with NASH, advanced fibrosis, and cirrhosis [67]. Normal ALT levels should therefore be taken with a grain of salt. One study proposed a stage-based approach that uses non-invasive scores alongside VCTE to risk-stratify patients with NAFLD and determine when to consider liver biopsy [68]. A more recent study by Davyduke et al. evaluated the impact of a "FIB-4 first" strategy to reduce the need for VCTE and hepatology referral [69]. Today, many investigational new drugs for NASH treatment are in phase III clinical trials, some of which might ultimately be approved by the U.S. Food and Drug Administration (FDA) as early as 2020.

We strongly believe that increased awareness of NAFLD and improved disease recognition among

Table 1 Noninvasive imaging assessment of NAFLD and advanced fibrosis

\begin{tabular}{|c|c|c|}
\hline Diagnostic modality & Advantages & Disadvantages \\
\hline$U^{a}$ & $\begin{array}{l}\text { - Noninvasive } \\
\text { - Inexpensive } \\
\text { - Widely available } \\
\text { - Fair accuracy in moderate to severe } \\
\text { hepatic steatosis }(\geq \mathrm{S} 2)^{\mathrm{a}}\end{array}$ & $\begin{array}{l}\downarrow \text { sensitivity when hepatic steatosis }<20-33 \%^{a} \\
\text { - Operator-dependent } \\
\text { - } \downarrow \text { accuracy in patients with chronic liver disease or obesity }\end{array}$ \\
\hline VCTE $\left(C A P^{a} \& L S M^{b}\right)$ & $\begin{array}{l}\text { - Noninvasive } \\
\text { - Inexpensive } \\
\text { - Widely available } \\
\text { - Reproducible } \\
\text { - Advanced fibrosis staging }\end{array}$ & $\begin{array}{l}\text { - Technical limitations in patients with ascites, morbid } \\
\text { obesity, or } \uparrow \text { chest wall fat } \\
\text { - Measurement failure }\end{array}$ \\
\hline MRI-PDFF $\&$ MRE ${ }^{b}$ & $\begin{array}{l}\text { - Noninvasive } \\
\text { - Quantification of hepatic steatosis }{ }^{a} \text { (helpful } \\
\text { in patients with } \downarrow \text { grade hepatic steatosis) } \\
\text { - Excellent reproducibility } \\
\text { - Advanced fibrosis staging }\end{array}$ & $\begin{array}{l}\text { - Expensive } \\
\text { - Small sample volume/not convenient for patients with } \\
\text { uneven fatty changes }{ }^{\mathrm{a}}\end{array}$ \\
\hline
\end{tabular}

${ }^{\mathrm{a}}$ steatosis assessment. ${ }^{\mathrm{b}}$ fibrosis assessment

Abbreviations: US Ultrasonography, VCTE Vibration-controlled transient elastography, CAP Controlled attenuation parameter, LSM liver Stiffness measurement, MRIPDFF Magnetic resonance imaging-proton density fat fraction, MRE Magnetic resonance elastography 
Table 2 Demographic- and serum-based biomarkers for fibrosis staging

\begin{tabular}{lll}
\hline Biomarker & Components & $\begin{array}{l}\text { Cut-offs to rule out/in } \\
\text { advanced fibrosis }\end{array}$ \\
\hline FIB-4 index [58] & Age, AST, ALT, and platelets & $<1.3>2.67$ \\
NAFLD fibrosis score & Age, BMI, IFG and diabetes, AST-to-ALT ratio, platelets, and albumin & $<-1.455$ \\
[58] & & $>0.676$ \\
$\begin{array}{l}\text { Enhanced liver fibrosis } \\
\text { test [59] }\end{array}$ & $\begin{array}{l}\text { Age, hyaluronic acid, aminoterminal propeptide of type III collagen, and tissue inhibitor of } \\
\text { matrix metalloproteinase 1 }\end{array}$ & $\geq 9.8$ \\
\hline
\end{tabular}

Abbreviations: BMI Body mass index, IFG Impaired fasting glucose, AST Aspartate aminotransferase, ALT Alanine aminotransferase, FIB Fibrosis index

NFS is calculated using the formula: NFS $=-1.675+0.037$ - age (years) $+0.094-\mathrm{BMI}\left(\mathrm{kg} / \mathrm{m}^{2}\right)+1.13 \times \mathrm{IFG} /$ diabetes $($ yes $=1$, no $=0)+0.99 \times \mathrm{AST} / \mathrm{ALT}$ ratio $-0.013 \times$ platelet count $\left(\times 10^{9} / \mathrm{l}\right)-0.66 \times$ albumin $(\mathrm{g} / \mathrm{dl})$. (https://nafldscore.com/)

FIB-4 is calculated using the formula: FIB-4 = Age (years) $\times$ AST $(\mathrm{U} / \mathrm{L}) /\left[\mathrm{PLT}\left(10^{9} / \mathrm{L}\right) \times \mathrm{ALT}^{1 / 2}(\mathrm{U} / \mathrm{L})\right]$ (https://www.hepatitisc.uw.edu/page/clinical-calculators/fib-4)

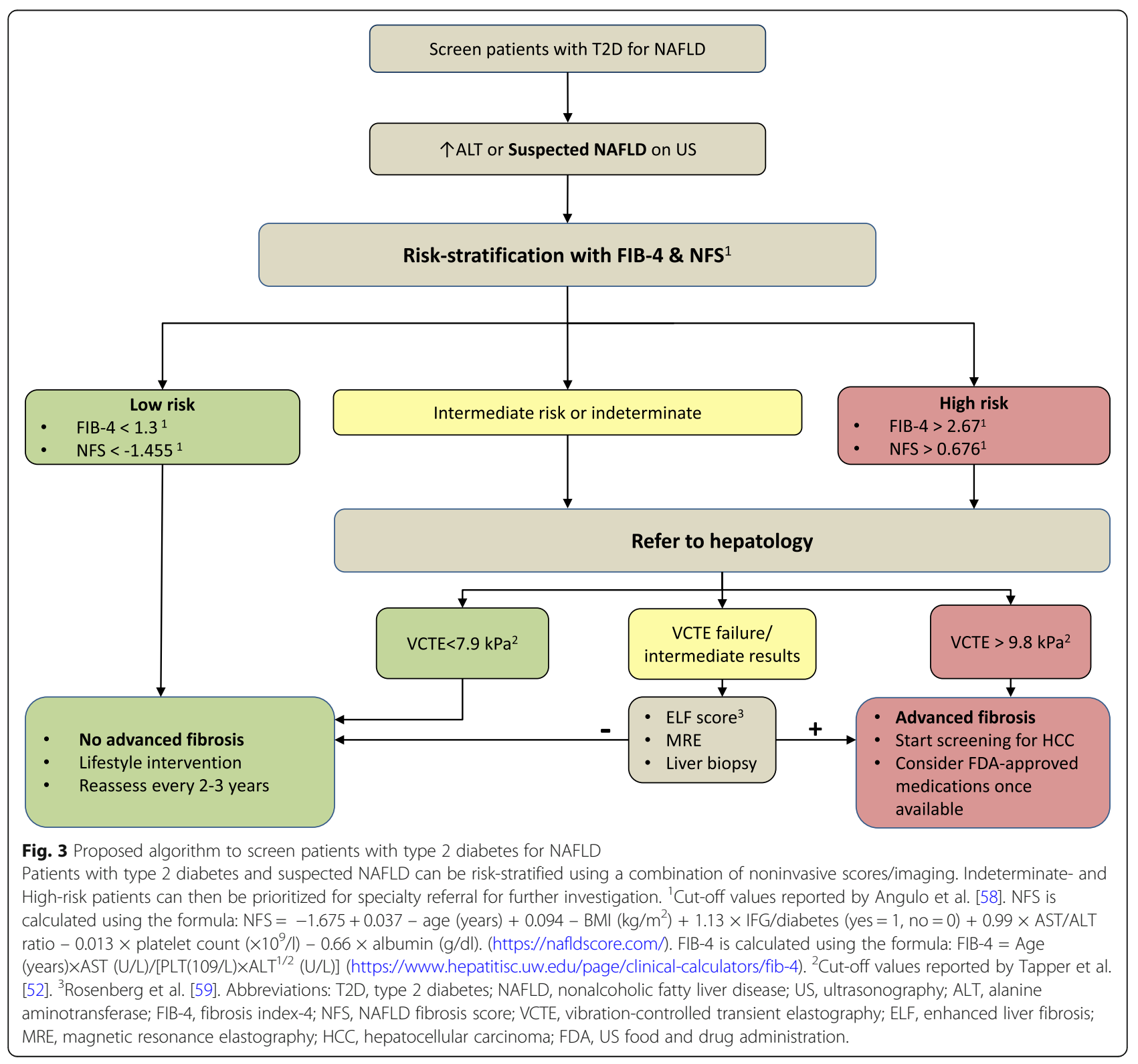


diabetologists would help in identifying patients with prediabetes and diabetes who might benefit from risk factor modification or emerging novel therapies to slow the progression of CVD and hepatic complications. Using validated risk scores like FIB-4 [64, 65] within electronic health records, similar to eGFR calculation, maybe a good initial step. In Fig. 3, we suggest an algorithm to aid diabetologists and primary care providers in screening patients with type 2 diabetes for NAFLD and advanced fibrosis. The cost-effectiveness of screening for NAFLD may still be debated; however, we believe proactive screening is better than passive waiting for fibrosis progression.

\section{Therapeutic approaches}

\section{Lifestyle intervention}

Lifestyle intervention with diet, exercise, and behavioral modification is the initial step in managing type 2 diabetes [70]. This also applies to patients with NAFLD [71]. Steatosis can be reduced by as little as $3-5 \%$ weight loss. On the other hand, 7-9\% weight loss is typically needed to reduce inflammation, while $10 \%$ is required to initiate fibrosis regression [72]. Although it is widely thought that sustainable weight loss through lifestyle modification is often difficult to achieve, a multidisciplinary approach to lifestyle intervention in patients with type 2 diabetes has been shown to induce weight loss that is both maintainable and clinically meaningful. We previously reported that $53 \%$ of participants in a realworld, multidisciplinary lifestyle intervention program who achieved an average of $\geq 7 \%$ weight loss at 1 year were able to maintain up to $9 \%$ weight loss at 5 years [73]. On the other hand, a Mediterranean eating pattern was shown to reduce hepatic steatosis and IR independent of weight loss in insulin-resistant individuals without diabetes but with biopsy-proven NAFLD [74]. Other strategies to induce weight loss, such as bariatric surgery and endoscopic bariatric procedures may be considered in NAFLD $[2,75]$. In patients with type 2 diabetes, bariatric surgery was shown to reduce body weight, HbA1c, insulin resistance, and has led to partial or complete diabetes remission in some cases [76, 77]. More recently, duodenal mucosal resurfacing (DMR), a novel and minimally invasive endoscopic procedure, improved glycemic and hepatic indices in patients with type 2 diabetes, which shows promise of possible benefits in patients with NAFLD [78]. More data are still needed regarding the long-term efficacy of bariatric surgery and DMR on histologic severity and disease progression in patients with NASH $[75,79]$.

\section{Diabetes pharmacotherapy for NAFLD treatment}

The dynamic association between NAFLD and hepatic IR has led to the experimentation of several diabetes medications for the treatment of NAFLD. These trials generated knowledge that may support future management paradigms for NAFLD in patients with diabetes, but further questions need to be answered.

\section{Metformin}

An adenosine monophosphate (AMP)-activated protein kinase (AMPK) activator; metformin is the first-line pharmacologic treatment for prediabetes and type 2 diabetes. Although several randomized controlled trials (RCTs) reported that metformin did not improve histologic features of NAFLD [80, 81], metformin may lower the risk of HCC in patients with diabetes [82]. In a recent translational study that included lung biopsies of humans with idiopathic pulmonary fibrosis (IPF) and a bleomycin mouse model (an experimental mouse model of lung fibrosis), AMPK activity was lower in fibrotic foci associated with active myofibroblasts. Moreover, the study reported that pharmacological activation of AMPK with metformin can reverse established fibrosis by facilitating deactivation and apoptosis of myofibroblasts [83]. In a more recent retrospective analysis of 191 patients with diabetes and biopsy-proven NASH and bridging fibrosis or compensated cirrhosis, metformin use was linked to lower risk of overall mortality and liver transplant (HR: 0.42; 95\% CI: 0.24-0.74, $p=0.003$ ) and HCC (sHR: $0.25 ; 95 \%$ CI: $0.11-0.58, p=0.001$ ) [84]. These findings may pave the way for studying AMPK activators in NAFLD, such as PXL770, which is being evaluated in a randomized clinical trial versus placebo to assess its effects on liver fat reduction after 12 weeks of treatment (ClinicalTrials.gov Identifier: NCT03763877). Metformin should be used with caution in patients with an estimated glomerular filtration rate (eGFR) $<45 \mathrm{~mL} / \mathrm{min}$, and possibly discontinued if eGFR drops $<30$.

\section{Glucagon-like Peptide-1 receptor agonists}

The glucagon-like peptide-1 (GLP-1) receptor agonist liraglutide was studied in a recent multicenter, double-blind, randomized, placebo-controlled phase two study in patients with NASH (the LEAN study) [85]. NASH resolution was observed in nine patients (39\%) in the liraglutide group in comparison to two patients (9\%) in the placebo group $(p=$ 0.019). Semaglutide, a longer-acting GLP-1 receptor agonist, is currently being studied in a larger sample size of 288 patients with NASH (ClinicalTrials.gov Identifier: NCT02970942). Semaglutide is also being studied in combination with other medications that inhibit hepatic DNL and affect bile acid-enterohepatic access (ClinicalTrials.gov Identifier: NCT03987074). There is conflicting evidence on risk of acute pancreatitis with the use of GLP-1 receptor agonists [86, 87]. Patients with type 2 diabetes and NASH should be made aware of these possible risks. 


\section{Thiazolidinediones}

In a study that randomized 247 non-diabetic patients with NASH to either $30 \mathrm{mg}$ of pioglitazone daily, $800 \mathrm{IU}$ of vitamin $\mathrm{E}$ daily, or placebo, pioglitazone was not superior to placebo in improving histologic features of $\mathrm{NASH}$ after 96 weeks of intervention. However, the study showed that pioglitazone use was associated with significant improvements in hepatic steatosis, ALT, and AST compared to placebo [88]. In a more recent RCT that randomized 101 patients with prediabetes or type 2 diabetes and biopsy-proven NASH to receive either 45 $\mathrm{mg}$ of pioglitazone daily or placebo for 72 weeks, $51 \%$ of patients in the pioglitazone arm had resolution of NASH and improvement in several histologic features, including liver fibrosis [89]. Currently, Pioglitazone is the only diabetes medication included in recent guidance from the AASLD to treat patients with biopsy-proven NASH with or without type 2 diabetes [2, 90]; however, it should be prescribed with caution given its safety profile (potential weight gain, and risk of bladder cancer, bone loss, and congestive heart failure) [91].

\section{Sodium-glucose co-transporter 2 inhibitors}

These medications inhibit glucose reabsorption in the proximal tubule, which leads to significant loss of glucose and calorie in the urine, resulting in improved insulin sensitivity, weight reduction, and potentially a reduction in liver fat content [92]. Previous reports linked remogliflozin to a $30-40 \%$ reduction in ALT levels in patients with abnormal baseline ALT [93]. canagliflozin [94] and dapagliflozin [95] also showed benefits in reducing serum aminotransferases. In a recent RCT that randomized 50 patients with type 2 diabetes and NAFLD to receive either standard diabetes care and empagliflozin or standard diabetes care only, liver fat, as measured by MRI-PDFF, decreased significantly in the empagliflozin arm compared to the comparative arm [96]. Further RCTs are needed to determine the effect of SGLT2 inhibitors on liver histology in NASH.

\section{Treatment considerations}

There is evidence, although not conclusive, that the use of insulin and oral insulin secretagogues, including sulfonylureas, may be associated with an increased risk of HCC in patients with type 2 diabetes $[97,98]$ possibly through insulin-mediated cancer cell proliferation [99]. Therefore, similar to the recent recommendation that CVD risk reduction should be taken into account when treating patients with type 2 diabetes, It is important to take into consideration the risk of NAFLD progression to HCC. When primary care providers see patients with type 2 diabetes and NAFLD being treated with metformin and glipizide, only it may be plausible to replace glipizide with a GLP-1 or SGLT-2 agent. This is important to consider, but caution is advised.

Finally, combination therapy is projected to be the future of NASH management, particularly in patients with co-existing type 2 diabetes. One attracting combination to improve NASH while reducing CVD risk may include low-dose pioglitazone with either a GLP-1 analogue or SGLT-2 inhibitor; however, future studies are needed to explore the efficacy of such combination [90].

\section{Tipping the scale-novel NASH therapies in the pipeline}

The quest for a NASH-specific treatment has been harnessing significant attention from federal and private funders as well as the pharmaceutical industry. As of August 15, 2019, there were 750 NAFLD trials registered on clinicaltrilas.gov. Earlier in 2019, results from the STELLAR-3, a phase three, randomized, double-blind, placebo-controlled study which evaluated the safety and efficacy of selonsertib, an apoptosis signal-regulating kinase 1 (ASK1) inhibitor showed no superiority to placebo in the primary endpoint of $a \geq 1$-stage histologic improvement in fibrosis without worsening of NASH in patients with bridging NASH-fibrosis (F3)(ClinicalTrials. gov Identifier: NCT03053050).

The farnesoid $\mathrm{X}$ receptor (FXR) agonist obeticholic acid (OCA) is the most advanced drug in the pipeline. FXR is a nuclear receptor with high expression in the liver and small intestine [100]. FXR naturally binds to bile acids [100], and they jointly regulate lipid/ glucose homeostasis, promote insulin sensitivity, and potentially modify liver fibrosis [101].

In phase two and three trials in patients with NASH and advanced fibrosis, OCA showed efficacy on fibrosis regression, paving the way for potential FDA approval by 2020 [102, 103]. In the phase 3 REGENERATE trial (NCT02548351), that randomized patients to receive OCA at $10 \mathrm{mg}$ or $25 \mathrm{mg}$ daily or placebo, the interim analysis at 18 months revealed significant improvement in fibrosis by one stage in patients on OCA $25 \mathrm{mg}$ daily compared to those in the placebo arm $(23.1 \%$ vs $11.9 \%$ $P=0.0002$ ) [103]. The fact that fibrosis improvement by one stage occurred in less than one-quarter of patients provides a strong rationale for the need for combination therapy with other drugs to increase efficacy.

Multiple investigational new drugs show promising potential in modifying $\mathrm{NASH}$ and fibrosis progression and are now in phase 3 clinical trials (Table 3 ). A dual peroxisome proliferator-activated receptor (PPAR) $\alpha / \delta$ agonist, elfibranor regulates glucose homeostasis and lipid metabolism and reduces inflammation, potentially modifying fibrosis [104] (NCT02704403). Resmetirom, a liver-directed thyroid hormone receptor- $\beta$ agonist, increases hepatic fat metabolism and reduces lipotoxicity, potentially improving NASH [105] (NCT03900429). Another agent in 
Table 3 NASH therapies in clinical trials

\begin{tabular}{|c|c|c|c|c|}
\hline & Description & Target & Phase & Duration \\
\hline Obeticholic acid & Semisynthetic bile acid & FXR agonist & \|\|$^{a}$ & 72 weeks \\
\hline Elafibranor & Small-molecule & Dual PPAR $a / \delta$ agonist & III & 72 weeks \\
\hline Resmetirom & Small-molecule & THR $\beta$ agonist & III & 52 weeks \\
\hline Aramchol & Synthetic FABAC & SCD-1 modulator & III & 52 weeks \\
\hline Cenicriviroc & Small-molecule & Dual CCR2/CCR5 antagonist & III & 48 weeks \\
\hline
\end{tabular}

Abbreviations: NASH Nonalcoholic steatohepatitis, FXR Farnesoid x receptor, PPAR Peroxisome proliferator-activated receptor, THR Thyroid hormone receptor, FABAC Fatty-acid/bile-acid conjugate, SCD Stearoyl-CoA desaturase, CCR C-c chemokine receptor

${ }^{\text {a }}$ Topline results demonstrated significant improvement in fibrosis by 1 stage in patients on OCA 25 mg daily compared to those in the placebo arm [103]

clinical trials is aramchol, a synthetic fatty-acid/bile-acid conjugate, which reduces hepatic fat through downregulation of stearoyl-CoA desaturase-1, a fatty acid synthetic enzyme in hepatocytes. The anti-fibrotic effect of aramchol stems from its upregulation of PPAR $\delta$ in hepatic stellate cells (HSCs), the primary fibrogenic cell type in the liver [106] (NCT04104321). Lastly, cenicriviroc, a dual c-c chemokine receptor $2 / 5$ antagonist which blocks intrahepatic macrophage trafficking and may confer antifibrotic effects through de-activation of HSCc [107, 108] (NCT03028740). These agents are currently being studied. If any deemed effective, an expected FDA-approval in late 2020 would be looming. Future studies should explore the efficacy of combined anti-fibrotic and anti-diabetic therapy in the management of patients with type 2 diabetes and NASH/fibrosis [90].

\section{Conclusions}

The prevalence of NASH among patients with type 2 diabetes is high, putting them at a significantly higher risk for developing end-stage liver disease, HCC, and CVD. Increased awareness about NASH and NASHrelated complications is warranted among diabetologists, especially with the prospective induction of NASHspecific therapies. We believe an interdisciplinary approach is needed for the care of patients with type 2 diabetes and NAFLD starting with early identification through noninvasive biomarkers and imaging modalities in the diabetes clinic to lifestyle modification and $\mathrm{NASH}$-specific therapy in the hepatology clinic.

\section{Abbreviations}

NAFLD: Nonalcoholic fatty liver disease; NAFL: Nonalcoholic fatty liver; NASH: Nonalcoholic steatohepatitis; HCC: Hepatocellular carcinoma; CVD: Cardiovascular disease; IR: Insulin resistance; FFAs: Free fatty acids; DNL: De novo lipogenesis; MetS: Metabolic syndrome; US: Ultrasonography; VCTE: Vibration controlled transient elastography; AASLD: American Association for the Study of Liver Diseases; MRI-PDFF: Magnetic resonance imaging-derived proton density fat fraction; LSM: Liver stiffness measurement; HSI: Hepatic steatosis index; FLI: Fatty liver index; NFS: NAFLD fibrosis score; AST: Aspartate aminotransferase; APRI: AST to platelet ratio; ALT: Alanine aminotransferase; DMR: Duodenal mucosal resurfacing; AMP: Adenosine monophosphate; AMPK: Activated protein kinase; RCTs: Randomized controlled trials; IPF: Idiopathic pulmonary fibrosis; GLP1: Glucagon-like peptide-1; ASK1: Apoptosis signal-regulating kinase 1; FXR: Farnesoid X receptor; OCA: Obeticholic acid; PPAR: Peroxisome proliferator-activated receptor; HSCs: Hepatic stellate cells

\section{Acknowledgments}

We thank Hannah Gardner for her help with illustrating Fig. 2. The gastrointestinal tract illustration in Fig. 2 was adapted from work available in public domain by Mariana Ruiz Villarreal https://jbo.wikipedia.org/ wiki/datnyvei:Digestive_system_without_labels.svg.

\section{Contributors}

Not applicable.

\section{Authors' contributions}

All authors searched the literature and wrote the manuscript. All authors reviewed and approved the final version of the manuscript.

Funding

The authors declare no source of funding for this manuscript.

\section{Availability of data and materials}

Data sharing is not applicable to this article as no data sets were generated or analyzed during the current study.

Ethics approval and consent to participate Not applicable.

\section{Competing interests}

S.T. has nothing to disclose. N.A. advises, is on the speakers' bureau for, and received grants from Gilead and Intercept. He advises and received grants from Allergan. He received grants from GENFIT, Madrigal, and Galmed. O.H. reports consultation to Abbott Nutrition, Gilead Inc. and Merck Sorono, grants from National Dairy Council, and own shares in Heathimation Inc. outside the submitted work.

\section{Author details}

${ }^{1}$ Research Division, Joslin Diabetes Center, 1 Joslin Place, Boston, MA 02215 USA. ${ }^{2}$ Department of Medicine, Harvard Medical School, Boston, MA 02215, USA. ${ }^{3}$ Texas Liver Institute, University of Texas (UT) Health, San Antonio, TX, USA.

Received: 13 February 2020 Accepted: 10 May 2020

Published online: 05 June 2020

References

1. Singh S, Allen AM, Wang Z, Prokop LJ, Murad MH, Loomba R. Fibrosis Progression in Nonalcoholic Fatty Liver vs Nonalcoholic Steatohepatitis: A Systematic Review and Meta-analysis of Paired-Biopsy Studies. Clin Gastroenterol Hepatol. 2015;13(4):643-54.e9.

2. Chalasani N, Younossi Z, Lavine JE, Charlton M, Cusi K, Rinella M, et al. The diagnosis and management of nonalcoholic fatty liver disease: practice guidance from the American Association for the Study of Liver Diseases. Hepatology. 2018;67(1):328-57.

3. Crespo M, Lappe S, Feldstein AE, Alkhouri N. Similarities and differences between pediatric and adult nonalcoholic fatty liver disease. Metabol Clin Exp. 2016:65(8):1161-71.

4. Masarone M, Federico A, Abenavoli L, Loguercio C, Persico M. Non alcoholic fatty liver: epidemiology and natural history. Rev Recent Clin Trials. 2014;9(3): 126-33. 
5. Lonardo A, Bellentani S, Argo CK, Ballestri S, Byrne CD, Caldwell SH, et al. Epidemiological modifiers of non-alcoholic fatty liver disease: focus on highrisk groups. Dig Liver Dis. 2015;47(12):997-1006.

6. Younossi ZM, Golabi P, de Avila L, Paik JM, Srishord M, Fukui N, et al. The global epidemiology of NAFLD and NASH in patients with type 2 diabetes: a systematic review and meta-analysis. J Hepatol. 2019;71(4):793-801.

7. Younossi Z, Tacke F, Arrese M, Chander Sharma B, Mostafa I, Bugianesi E, et al. Global perspectives on nonalcoholic fatty liver disease and nonalcoholic Steatohepatitis. Hepatology. 2019;69(6):2672-82.

8. Hassan K, Bhalla V, El Regal ME, HH AK. Nonalcoholic fatty liver disease: a comprehensive review of a growing epidemic. World J Gastroenterol. 2014; 20(34):12082-101.

9. Estes C, Razavi H, Loomba R, Younossi Z, Sanyal AJ. Modeling the epidemic of nonalcoholic fatty liver disease demonstrates an exponential increase in burden of disease. Hepatology. 2018;67(1):123-33.

10. Calzadilla Bertot $L$, Adams $L A$. The natural course of non-alcoholic fatty liver disease. Int J Mol Sci. 2016;17(5):774

11. Caldwell SH, Crespo DM. The spectrum expanded: cryptogenic cirrhosis and the natural history of non-alcoholic fatty liver diseasePowell EE, Cooksley WGE, Hanson R, Searle J, Halliday JW, Powell LW. The natural history of nonalcoholic steatohepatitis: a follow-up study of forty-two patients for up to 21 years [Hepatology 1990; 11: 74-80]. J Hepatol. 2004;40(4):578-84.

12. Charlton MR, Burns JM, Pedersen RA, Watt KD, Heimbach JK, Dierkhising RA. Frequency and outcomes of liver transplantation for nonalcoholic steatohepatitis in the United States. Gastroenterology. 2011;141(4):1249-53.

13. Doycheva I, Issa D, Watt KD, Lopez R, Rifai G, Alkhouri N. Nonalcoholic steatohepatitis is the most rapidly increasing indication for liver transplantation in young adults in the United States. J Clin Gastroenterol. 2018:52(4):339-46.

14. Noureddin M, Vipani A, Bresee C, Todo T, Kim IK, Alkhouri N, et al. NASH leading cause of liver transplant in women: updated analysis of indications for liver transplant and ethnic and gender variances. Am J Gastroenterol. 2018;113(11):1649-59.

15. Alexander $\mathrm{M}$, Loomis AK, van der Lei J, Duarte-Salles T, Prieto-Alhambra $\mathrm{D}$ Ansell $\mathrm{D}$, et al. Non-alcoholic fatty liver disease and risk of incident acute myocardial infarction and stroke: findings from matched cohort study of 18 million European adults. BMJ. 2019;367:15367.

16. Targher G, Byrne CD, Lonardo A, Zoppini G, Barbui C. Non-alcoholic fatty liver disease and risk of incident cardiovascular disease: a meta-analysis. J Hepatol. 2016;65(3):589-600.

17. Luo J, Xu L, Li J, Zhao S. Nonalcoholic fatty liver disease as a potential risk factor of cardiovascular disease. Eur J Gastroenterol Hepatol. 2015;27(3):1939.

18. Yamaguchi K, Yang L, McCall S, Huang J, Yu XX, Pandey SK, et al. Inhibiting triglyceride synthesis improves hepatic steatosis but exacerbates liver damage and fibrosis in obese mice with nonalcoholic steatohepatitis. Hepatology. 2007;45(6):1366-74.

19. Greenfield V, Cheung O, Sanyal AJ. Recent advances in nonalcholic fatty liver disease. Curr Opin Gastroenterol. 2008;24(3):320-7.

20. Lambert JE, Ramos-Roman MA, Browning JD, Parks EJ. Increased de novo lipogenesis is a distinct characteristic of individuals with nonalcoholic fatty liver disease. Gastroenterology. 2014;146(3):726-35.

21. Marra F, Bertolani C. Adipokines in liver diseases. Hepatology. 2009;50(3): 957-69.

22. Robertson RP, Harmon J, Tran POT, Poitout V. B-Cell Glucose Toxicity, Lipotoxicity, and Chronic Oxidative Stress in Type 2 Diabetes. Diabetes. 2004;53(suppl 1):S119-S24.

23. Gastaldelli A, Cusi K. From NASH to diabetes and from diabetes to NASH: mechanisms and treatment options. JHEP Rep. 2019;1(4):312-28.

24. Softic S, Meyer JG, Wang G-X, Gupta MK, Batista TM, Lauritzen HPMM, et al. Dietary Sugars Alter Hepatic Fatty Acid Oxidation via Transcriptional and Post-translational Modifications of Mitochondrial Proteins. Cell Metab. 2019; 30(4):735-53.e4.

25. Tilg H, Moschen AR. Evolution of inflammation in nonalcoholic fatty liver disease: the multiple parallel hits hypothesis. Hepatology. 2010;52(5):1836-46.

26. Miele L, Valenza V, La Torre G, Montalto M, Cammarota G, Ricci R, et al. Increased intestinal permeability and tight junction alterations in nonalcoholic fatty liver disease. Hepatology. 2009:49(6):1877-87.

27. Kakisaka K, Cazanave SC, Werneburg NW, Razumilava N, Mertens JC, Bronk SF, et al. A hedgehog survival pathway in 'undead'lipotoxic hepatocytes. J Hepatol. 2012;57(4):844-51.
28. Ekstedt M, Hagström H, Nasr P, Fredrikson M, Stål P, Kechagias S, et al. Fibrosis stage is the strongest predictor for disease-specific mortality in NAFLD after up to 33 years of follow-up. Hepatology. 2015;61(5):1547-54.

29. Cani PD, Amar J, Iglesias MA, Poggi M, Knauf C, Bastelica D, et al. Metabolic Endotoxemia initiates obesity and insulin resistance. Diabetes. 2007;56(7): $1761-72$.

30. Everard A, Cani PD. Diabetes, obesity and gut microbiota. Best Pract Res Clin Gastroenterol. 2013;27(1):73-83.

31. Boulange CL, Neves AL, Chilloux J, Nicholson JK, Dumas ME. Impact of the gut microbiota on inflammation, obesity, and metabolic disease. Genome Med. 2016;8(1):42.

32. Kim $\mathrm{CH}$, Younossi ZM. Nonalcoholic fatty liver disease: a manifestation of the metabolic syndrome. Cleve Clin J Med. 2008;75(10):721-8.

33. Gastaldelli A. Fatty liver disease: the hepatic manifestation of metabolic syndrome. Hypertens Res. 2010:33(6):546-7.

34. European Association for the Study of the L, European Association for the Study of D, European Association for the Study of O. EASL-EASD-EASO clinical practice guidelines for the management of non-alcoholic fatty liver disease. J Hepatol. 2016:64(6):1388-402.

35. Jiang ZG, Tapper EB. Cost saving or cost effective? Unanswered questions in the screening of patients with nonalcoholic fatty liver disease. Hepatol Commun. 2019;3(10):1293-5.

36. Garg K, Brackett S, Hirsch IB, Garg SK. NAFLD/NASH and Diabetes. Diabetes Technol Ther. 2020;22(S1):S-174-S-86.

37. Alexander M, Loomis AK, Fairburn-Beech J, van der Lei J, Duarte-Salles T, Prieto-Alhambra D, et al. Real-world data reveal a diagnostic gap in nonalcoholic fatty liver disease. BMC Med. 2018;16(1):130.

38. Singh A, Le P, Lopez R, Alkhouri N. The utility of noninvasive scores in assessing the prevalence of nonalcoholic fatty liver disease and advanced fibrosis in type 1 diabetic patients. Hepatol Int. 2018;12(1):37-43.

39. Mottalib A, Tomah S, Hafida S, Elseaidy T, Kasetty M, Ashrafzadeh S, et al. Intensive multidisciplinary weight management in patients with type 1 diabetes and obesity: a one-year retrospective matched cohort study. Diabetes Obes Metab. 2019;21(1):37-42.

40. Li Y, Wang J, Tang Y, Han X, Liu B, Hu H, et al. Bidirectional association between nonalcoholic fatty liver disease and type 2 diabetes in Chinese population: evidence from the Dongfeng-Tongji cohort study. PLoS One. 2017:12(3):e0174291.

41. Ludwig J, Viggiano TR, Mcgill DB, Oh B. Nonalcoholic steatohepatitis: Mayo Clinic experiences with a hitherto unnamed disease. Mayo Clin Proc. 1980; 55(7):434-8.

42. Boyle M, Masson S, Anstee QM. The bidirectional impacts of alcohol consumption and the metabolic syndrome: cofactors for progressive fatty liver disease. J Hepatol. 2018;68(2):251-67.

43. Cusi K. Time to Include Nonalcoholic Steatohepatitis in the Management of Patients With Type 2 Diabetes. Diabetes Care. 2020;43(2):275-9.

44. Association AD. 4. Comprehensive Medical Evaluation and Assessment of Comorbidities: Standards of Medical Care in Diabetes-2019. Diabetes care. 2019:42(Suppl 1):S34.

45. Al Knawy B, Shiffman M. Percutaneous liver biopsy in clinical practice. Liver Int. 2007:27(9):1166-73.

46. Anstee QM, Targher G, Day CP. Progression of NAFLD to diabetes mellitus, cardiovascular disease or cirrhosis. Nat Rev Gastroenterol Hepatol. 2013; 10(6):330-44.

47. Dasarathy S, Dasarathy J, Khiyami A, Joseph R, Lopez R, McCullough AJ. Validity of real time ultrasound in the diagnosis of hepatic steatosis: a prospective study. J Hepatol. 2009;51(6):1061-7.

48. Caussy C, Reeder SB, Sirlin CB, Loomba R. Noninvasive, quantitative assessment of liver fat by MRI-PDFF as an endpoint in NASH trials. Hepatology. 2018;68(2):763-72.

49. Siddiqui MS, Vuppalanchi R, Van Natta ML, Hallinan E, Kowdley KV, Abdelmalek M, et al. Vibration-controlled transient elastography to assess fibrosis and steatosis in patients with nonalcoholic fatty liver disease. Clin Gastroenterol Hepatol. 2019;17(1):156-63. e2.

50. Castera L, Forns X, Alberti A. Non-invasive evaluation of liver fibrosis using transient elastography. J Hepatol. 2008:48(5):835-47.

51. Wilder J, Patel K. The clinical utility of FibroScan ${ }^{\oplus}$ as a noninvasive diagnostic test for liver disease. Med Devices (Auckland, NZ). 2014;7:107.

52. Tapper EB, Challies T, Nasser I, Afdhal NH, Lai M. The performance of vibration controlled transient Elastography in a US cohort of patients with nonalcoholic fatty liver disease. Am J Gastroenterol. 2016;111(5):677-84. 
53. Mann J, Reeves HL, Feldstein AE. Liquid biopsy for liver diseases. Gut. 2018 67(12):2204-12.

54. Wong W-S, Adams LA, de Lédinghen V, Wong GL-H, Sookoian S. Noninvasive biomarkers in NAFLD and NASH - current progress and future promise. Nat Rev Gastroenterol Hepatol. 2018;15(8):461-78.

55. Anstee QM, Lawitz EJ, Alkhouri N, Wong WWS, Romero-Gomez M, Okanoue T, et al. Noninvasive tests accurately identify advanced fibrosis due to NASH: baseline data from the STELLAR trials. Hepatology. 2019;70:1521-30.

56. Lee J-H, Kim D, Kim HJ, Lee C-H, Yang Jl, Kim W, et al. Hepatic steatosis index: a simple screening tool reflecting nonalcoholic fatty liver disease. Dig Liver Dis. 2010;42(7):503-8.

57. Bedogni G, Bellentani S, Miglioli L, Masutti F, Passalacqua M, Castiglione A, et al. The fatty liver index: a simple and accurate predictor of hepatic steatosis in the general population. BMC Gastroenterol. 2006;6(1):33.

58. Angulo $P$, Bugianesi $E$, Bjornsson ES, Charatcharoenwitthaya $P$, Mills PR Barrera F, et al. Simple noninvasive systems predict long-term outcomes of patients with nonalcoholic fatty liver disease. Gastroenterology. 2013;145(4): 782-9.e4.

59. Rosenberg WM, Voelker M, Thiel R, Becka M, Burt A, Schuppan D, et al. Serum markers detect the presence of liver fibrosis: a cohort study. Gastroenterology. 2004;127(6):1704-13.

60. Sterling RK, Lissen E, Clumeck N, Sola R, Correa MC, Montaner J, et al. Development of a simple noninvasive index to predict significant fibrosis in patients with HIV/HCV coinfection. Hepatology. 2006;43(6):1317-25.

61. Angulo P, Hui JM, Marchesini G, Bugianesi E, George J, Farrell GC, et al. The NAFLD fibrosis score: a noninvasive system that identifies liver fibrosis in patients with NAFLD. Hepatology. 2007;45(4):846-54.

62. Lin ZH, Xin YN, Dong QJ, Wang Q, Jiang XJ, Zhan SH, et al. Performance of the aspartate aminotransferase-to-platelet ratio index for the staging of hepatitis Crelated fibrosis: an updated meta-analysis. Hepatology. 2011;53(3):726-36.

63. Singh A, Gosai F, Siddiqui MT, Gupta M, Lopez R, Lawitz E, et al. Accuracy of Noninvasive Fibrosis Scores to Detect Advanced Fibrosis in Patients With Type-2 Diabetes With Biopsy-proven Nonalcoholic Fatty Liver Disease. J Clin Gastroenterol. 2020. https://doi.org/10.1097/MCG.0000000000001339.

64. Shah AG, Lydecker A, Murray K, Tetri BN, Contos MJ, Sanyal AJ, et al. Use of the FIB4 index for non-invasive evaluation of fibrosis in nonalcoholic fatty liver disease. Clin Gastroenterol Hepatol. 2009;7(10):1104.

65. Siddiqui MS, Yamada G, Vuppalanchi R, Van Natta M, Loomba R, Guy C, et al. Diagnostic accuracy of noninvasive fibrosis models to detect change in fibrosis stage. Clin Gastroenterol Hepatol. 2019;17(9):1877-85 e5.

66. Pandyarajan V, Gish RG, Alkhouri N, Noureddin M. Screening for nonalcoholic fatty liver disease in the primary care clinic. Gastroenterol Hepatol (N Y). 2019;15(7):357-65.

67. Gawrieh S, Wilson LA, Cummings OW, Clark JM, Loomba R, Hameed B, et al. Histologic Findings of Advanced Fibrosis and Cirrhosis in Patients With Nonalcoholic Fatty Liver Disease Who Have Normal Aminotransferase Levels. Am J Gastroenterol. 2019;114(10):1626-35.

68. Rinella ME, Sanyal AJ. Management of NAFLD: a stage-based approach. Nat Rev Gastroenterol Hepatol. 2016;13(4):196-205.

69. Davyduke T, Tandon P, Al-Karaghouli M, Abraldes JG, Ma MM. Impact of implementing a "FIB-4 first" strategy on a pathway for patients with NAFLD referred from primary care. Hepatol Commun. 2019;3(10):1322-33.

70. Association AD. 4. Lifestyle management: Standards of medical care in diabetes-2018. Diabetes Care. 2018;41(Supplement 1):S38-50.

71. Gerber L, Otgonsuren M, Mishra A, Escheik C, Birerdinc A, Stepanova M, et al. Non-alcoholic fatty liver disease (NAFLD) is associated with low level of physical activity: a population-based study. Aliment Pharmacol Ther. 2012;36(8):772-81.

72. Lassailly G, Caiazzo R, Pattou F, Mathurin P. Perspectives on treatment for nonalcoholic Steatohepatitis. Gastroenterology. 2016;150(8):1835-48.

73. Hamdy O, Mottalib A, Morsi A, El-Sayed N, Goebel-Fabbri A, Arathuzik G, et al. Long-term effect of intensive lifestyle intervention on cardiovascular risk factors in patients with diabetes in real-world clinical practice: a 5-year longitudinal study. BMJ Open Diabetes Res Care. 2017;5(1):e000259.

74. Ryan MC, Itsiopoulos C, Thodis T, Ward G, Trost N, Hofferberth S, et al. The Mediterranean diet improves hepatic steatosis and insulin sensitivity in individuals with non-alcoholic fatty liver disease. J Hepatol. 2013;59(1):138-43.

75. Lee Y, Doumouras AG, Yu J, Brar K, Banfield L, Gmora S, et al. Complete resolution of nonalcoholic fatty liver disease after bariatric surgery: a systematic review and meta-analysis. Clin Gastroenterol Hepatol. 2019;17(6): 1040-60. e11.
76. Batterham RL, Cummings DE. Mechanisms of diabetes improvement following bariatric/metabolic surgery. Diabetes Care. 2016;39(6):893-901.

77. Mulla CM, Middelbeek RJW, Patti ME. Mechanisms of weight loss and improved metabolism following bariatric surgery. Ann N Y Acad Sci. 2018; 1411(1):53-64.

78. van Baar AC, Beuers U, Wong K, Haidry R, Costamagna G, Hafedi A, et al. Endoscopic duodenal mucosal resurfacing improves glycaemic and hepatic indices in type 2 diabetes: 6-month multicentre results. JHEP Rep. 2019;1(6):429-37.

79. Klebanoff MJ, Corey KE, Samur S, Choi JG, Kaplan LM, Chhatwal J, et al. Cost-effectiveness Analysis of Bariatric Surgery for Patients With Nonalcoholic Steatohepatitis CirrhosisCost-effectiveness of Bariatric Surgery for Patients With NASH CirrhosisCost-effectiveness of Bariatric Surgery for Patients With NASH Cirrhosis. JAMA Network Open. 2019;2(2):e190047 -e.

80. Lavine JE, Schwimmer JB, Van Natta ML, Molleston JP, Murray KF, Rosenthal $P$, et al. Effect of vitamin $E$ or metformin for treatment of nonalcoholic fatty liver disease in children and adolescents: the TONIC randomized controlled trial. Jama. 2011:305(16):1659-68.

81. Rakoski M, Singal A, Rogers $M$, Conjeevaram H. Meta-analysis: insulin sensitizers for the treatment of non-alcoholic steatohepatitis. Aliment Pharmacol Ther. 2010;32(10):1211-21.

82. Chen H-P, Shieh J-J, Chang C-C, Chen T-T, Lin J-T, Wu M-S, et al. Metformin decreases hepatocellular carcinoma risk in a dose-dependent manner: population-based and in vitro studies. Gut. 2013;62(4):606-15.

83. Rangarajan S, Bone N, Zmijewska A, Jiang S, Park D, Bernard K, et al. Metformin reverses established lung fibrosis in a bleomycin model. Nat Med. 2018;24(8):1121-7.

84. Vilar-Gomez E, Vuppalanchi R, Desai A, Gawrieh S, Ghabril M, Saxena R, et al. Long-term metformin use may improve clinical outcomes in diabetic patients with non-alcoholic steatohepatitis and bridging fibrosis or compensated cirrhosis. Aliment Pharmacol Ther. 2019;50:317-28.

85. Armstrong MJ, Gaunt P, Aithal GP, Barton D, Hull D, Parker R, et al. Liraglutide safety and efficacy in patients with non-alcoholic steatohepatitis (LEAN): a multicentre, double-blind, randomised, placebo-controlled phase 2 study. Lancet. 2016;387(10019):679-90.

86. Singh S, Chang H-Y, Richards TM, Weiner JP, Clark JM, Segal JB. Glucagonlike peptide 1-based therapies and risk of hospitalization for acute pancreatitis in type 2 diabetes mellitus: a population-based matched case-control study. JAMA Intern Med. 2013;173(7):534-9.

87. Abd El Aziz M, Cahyadi O, Meier JJ, Schmidt WE, Nauck MA. Incretin-based glucose-lowering medications and the risk of acute pancreatitis and malignancies: a meta-analysis based on cardiovascular outcomes trials. Diabetes Obes Metab. 2020;22(4):699-704.

88. Sanyal AJ, Chalasani N, Kowdley KV, McCullough A, Diehl AM, Bass NM, et al. Pioglitazone, vitamin E, or placebo for nonalcoholic steatohepatitis. N Engl J Med. 2010;362(18):1675-85.

89. Cusi K, Orsak B, Bril F, Lomonaco R, Hecht J, Ortiz-Lopez C, et al. Long-term pioglitazone treatment for patients with nonalcoholic Steatohepatitis and Prediabetes or type 2 diabetes mellitus: a randomized trial. Ann Intern Med. 2016;165(5):305-15.

90. Cusi K. A diabetologist's perspective of non-alcoholic steatohepatitis (NASH): Knowledge gaps and future directions. Liver Int. 2020;40(S1):82-8.

91. Shah P, Mudaliar S. Pioglitazone: side effect and safety profile. Expert Opin Drug Saf. 2010;9(2):347-54.

92. Mudaliar S, Polidori D, Zambrowicz B, Henry RR. Sodium-glucose cotransporter inhibitors: effects on renal and intestinal glucose transport: from bench to bedside. Diabetes Care. 2015;38(12):2344-53.

93. Wilkison W, Cheatham B, Walker S. Remogliflozin etabonate reduces insulin resistance and liver function enzymes: role for treatment of NASH. J Hepatol. 2015;62(Suppl 2):S211-S2.

94. Lavalle-González F, Januszewicz A, Davidson J, Tong C, Qiu R, Canovatchel W, et al. Efficacy and safety of canagliflozin compared with placebo and sitagliptin in patients with type 2 diabetes on background metformin monotherapy: a randomised trial. Diabetologia. 2013;56(12):2582-92.

95. Bailey CJ, Gross JL, Pieters A, Bastien A, List JF. Effect of dapagliflozin in patients with type 2 diabetes who have inadequate glycaemic control with metformin: a randomised, double-blind, placebo-controlled trial. Lancet. 2010;375(9733):2223-33.

96. Kuchay MS, Krishan S, Mishra SK, Farooqui KJ, Singh MK, Wasir JS, et al. Effect of empagliflozin on liver fat in patients with type 2 diabetes and nonalcoholic fatty liver disease: a randomized controlled trial (E-LIFT trial). Diabetes Care. 2018;41(8):1801-8. 
97. Singh S, Singh PP, Singh AG, Murad MH, Sanchez W. Anti-diabetic medications and the risk of hepatocellular cancer: a systematic review and meta-analysis. Am J Gastroenterol. 2013;108(6):881-91 quiz 92.

98. Chang C-H, Lin J-W, Wu L-C, Lai M-S, Chuang L-M. Oral insulin secretagogues, insulin, and cancer risk in type 2 diabetes mellitus. J Clin Endocrinol Metab. 2012;97(7):E1170-E5.

99. Pollak M. Insulin and insulin-like growth factor signalling in neoplasia. Nat Rev Cancer. 2008;8(12):915-28.

100. Ali AH, Carey EJ, Lindor KD. Recent advances in the development of farnesoid X receptor agonists. Ann Transl Med. 2015;3(1):5.

101. Carr RM, Reid AE. FXR agonists as therapeutic agents for non-alcoholic fatty liver disease. Curr Atheroscler Rep. 2015;17(4):500.

102. Neuschwander-Tetri BA, Loomba R, Sanyal AJ, Lavine JE, Van Natta ML, Abdelmalek MF, et al. Farnesoid $\mathrm{X}$ nuclear receptor ligand obeticholic acid for non-cirrhotic, non-alcoholic steatohepatitis (FLINT): a multicentre, randomised, placebo-controlled trial. Lancet. 2015;385(9972):956-65.

103. Younossi Z, Ratziu V, Loomba R, Rinella M, Anstee QM, Goodman Z, et al. Positive Results from REGENERATE: A Phase 3 International, Randomized, Placebo-Controlled Study Evaluating Obeticholic Acid Treatment for NASH. Hepatology. 2019;70(1):Abstract GS-06.

104. Ratziu V, Harrison SA, Francque S, Bedossa P, Lehert P, Serfaty L, et al. Elafibranor, an agonist of the peroxisome proliferator- activated receptor$a$ and $-\delta$, induces resolution of nonalcoholic steatohepatitis without fibrosis worsening. Gastroenterology. 2016;150(5):1147-59 e5.

105. Harrison SA, Bashir MR, Guy CD, Zhou R, Moylan CA, Frias JP, et al. Resmetirom (MGL-3196) for the treatment of non-alcoholic steatohepatitis: a multicentre, randomised, double-blind, placebo-controlled, phase 2 trial. Lancet. 2019:394(10213):2012-24.

106. Allen B, Mato JM, Craig A, Fernandez-Ramos D, Lopitz-Otsoa F, Hayardeny L, et al. Aramchol Downregulates SCD1 and induces PPARy in hepatic stellate cells to attenuate cellular activation and Fibrogenesis. 2018.

107. Friedman SL, Ratziu V, Harrison SA, Abdelmalek MF, Aithal GP, Caballeria J, et al. A randomized, placebo-controlled trial of cenicriviroc for treatment of nonalcoholic steatohepatitis with fibrosis. Hepatology. 2018;67(5):1754-67.

108. Kruger AJ, Fuchs BC, Masia R, Holmes JA, Salloum S, Sojoodi M, et al. Prolonged cenicriviroc therapy reduces hepatic fibrosis despite steatohepatitis in a diet-induced mouse model of nonalcoholic steatohepatitis. Hepatol Commun. 2018;2(5):529-45.

\section{Publisher's Note}

Springer Nature remains neutral with regard to jurisdictional claims in published maps and institutional affiliations.

Ready to submit your research? Choose BMC and benefit from:

- fast, convenient online submission

- thorough peer review by experienced researchers in your field

- rapid publication on acceptance

- support for research data, including large and complex data types

- gold Open Access which fosters wider collaboration and increased citations

- maximum visibility for your research: over $100 \mathrm{M}$ website views per year

At $\mathrm{BMC}$, research is always in progress.

Learn more biomedcentral.com/submissions 